\title{
Systemic arteriovenous fistulae for end-stage cyanosis after cavopulmonary connection: A useful bridge to transplantation
}

\author{
Edward J. Hickey, MD, Abdullah A. Alghamdi, MD, Maryam Elmi, BSc, Khalid S. Al-Najashi, MD, \\ Glen S. Van Arsdell, Christopher A. Caldarone, MD, John Coles, MD, and William G. Williams, MD
}

Objective: Intractable cyanosis after partial or complete cavopulmonary connection may rarely be managed by creating a systemic arteriovenous fistula. We investigated the long-term performance of arteriovenous fistulae.

\begin{abstract}
Methods: All 21 patients who received an arteriovenous fistula at The Hospital for Sick Children since the 1950s were investigated using parametric competing risk techniques. Primary arteriovenous fistula indication was (1) suboptimal pulmonary blood flow $(\mathrm{N}=15)$ or $(2)$ pulmonary shunting via pulmonary arteriovenous malformations $(\mathrm{N}=6$ ). Arteriovenous fistula longevity was determined by time to "occlusion" (absence of arteriovenous fistula flow via surgical ligation or spontaneous occlusion).
\end{abstract}

Results: All 21 patients had previously undergone second-stage palliation (Glenn shunt $=13$; bidirectional shunt $=9)$. Five patients had undergone Fontan completion. Death in the presence of a functioning arteriovenous fistula occurred in 5 patients. Patients with bidirectional shunts had a significantly higher risk of death with a functioning arteriovenous fistula in situ $(P=.04)$. High hemoglobin concentrations were associated with best outcome, and levels less than $170 \mathrm{~g} / \mathrm{L}$ were associated with a high risk of death despite a functioning arteriovenous fistula $(P<$ $.01)$. Arteriovenous fistula occlusion occurred in 10 patients. Earlier occlusion was associated with previous Fontan completion $(P=.02)$ and pulmonary arteriovenous malformations $(P=.03)$. Surgical ligation during cardiac transplantation was the cause of occlusion in 7 patients. In these 7 patients, the arteriovenous fistula functioned for a median of 4.8 years. After transplantation, survival was $67 \% \pm 19 \%$ at 5 years. Overall survival was $73 \% \pm$ $10 \% 15$ years after receiving an arteriovenous fistula (longest survival, 27.3 years).

Conclusion: In patients with adequate hematocrit, arteriovenous fistula offers an effective bridge to transplantation when a high-risk Fontan procedure is deferred. Performance is best after unidirectional cavopulmonary connection and worse in the presence of pulmonary arteriovenous malformations. Survival is $75 \%$ at 15 years, despite being considered end stage. (J Thorac Cardiovasc Surg 2010;139:128-34)

Supplemental material is available online.

Intractable cyanosis after partial or complete cavopulmonary connection may rarely be managed by creating a systemic arteriovenous fistula (AVF). The logic behind this strategy is to improve systemic oxygenation by augmenting cavopulmonary shunt flow. ${ }^{1}$ However, the long-term consequences of this end-stage palliative approach are not known.

Progressive cyanosis after cavopulmonary connection includes 2 distinct mechanisms. In the first situation, total cavopulmonary shunt flow gradually declines, leading to

From the Departments of Pediatrics and Surgery, Division of Cardiovascular Surgery, The Hospital for Sick Children, Toronto, Ontario, Canada.

Received for publication Jan 22, 2008; revisions received Oct 20, 2008; accepted for publication Nov 23, 2008; available ahead of print Nov 18, 2009.

Address for reprints: William G. Williams, MD, The Hospital for Sick Children, 555 University Avenue, Toronto, Ontario, Canada M5 G 1X8 (E-mail: bill.williams@ sickkids.ca)

0022-5223/\$36.00

Copyright (c) 2010 by The American Association for Thoracic Surgery doi: $10.1016 /$ j.jtcvs.2008.11.074 sluggish pulmonary perfusion and poor oxygenation. ${ }^{1,2}$ The process may be exacerbated by poor ventricular performance. The aim of AVF in this scenario is to augment total pulmonary flow by increasing the superior vena caval pressure within the cavopulmonary shunt.

In the second situation, intrapulmonary arteriovenous malformations develop as a consequence of cavopulmonary shunt physiology and the interruption of hepatopulmonary blood flow. ${ }^{3,4}$ Right-to-left shunting within the lung then leads to impaired oxygenation and systemic cyanosis. In this scenario, creating an AVF to augment pulmonary perfusion aims to compensate for the proportion of pulmonary blood flow shunted through pulmonary arteriovenous malformations. In addition, reestablishing first-pass pulmonary metabolism of hepatic effluent may restore beneficial pulmonary microvascular effects of a putative "hepatic factor.,"

We investigated our institutional experience with the use of $\mathrm{AVF}$ as a method of palliating failing cavopulmonary shunts. In particular, we aimed to (1) determine long-term outcomes for AVF recipients, (2) investigate patient-specific features associated with AVF performance, and (3) determine whether the underlying indication for AVF contributes to its long-term function. 


\section{Abbreviation and Acronym}

$\mathrm{AVF}=$ arteriovenous fistula

\section{MATERIALS AND METHODS}

All 21 patients who underwent surgical creation of an AVF at The Hospital for Sick Children between 1978 and 2007 were included in this retrospective investigation. To our knowledge, these include all such cases since the introduction of open cardiac surgical procedures at The Hospital for Sick Children in the late 1950s. Institutional ethics board approval was obtained, but a waiver of consent was issued because of the retrospective nature and because patients were not contacted directly.

The operative technique has been described. ${ }^{2}$ Briefly, under general anesthesia an incision is made $1 \mathrm{~cm}$ below and parallel to the lateral half of the clavicle. The pectoralis major muscle is split, and the axillary neurovascular bundle is identified. After systemic heparinization, a side-to-side anastomosis ( $\sim 6-7 \mathrm{~mm}$ in length) is constructed between the axillary artery and vein. The distal axillary vein is then ligated to yield a functional side-to-end arteriovenous anastomosis. Postoperative anticoagulation is not given.

The primary clinical indication for AVF creation was classified as 1) cyanosis due to pulmonary shunting via arteriovenous malformations or 2) cyanosis in the absence of arteriovenous malformations, presumed due to inadequate overall cavopulmonary shunt flow and oxygenation. Pulmonary arteriovenous malformations were confirmed preoperatively by angiography. ${ }^{3}$ In the pre-Fontan era, AVF was pursued because the only alternative was transplantation. Otherwise, the decision to undertake AVF was considered because the Fontan procedure had failed or subsequent Fontan completion or cardiac transplantation was considered an excessive risk for the patient.

A functioning AVF was defined as being patent (including at the point of death). Therefore, its therapeutic longevity, or durability, is represented by the duration of its patency: from the point of creation until the time of its occlusion. The mechanism of occlusion may be spontaneous occlusion or surgical division/ligation. Follow-up review of patients' records was undertaken in 2007 (mean of 11 years from the point of AVF, up to a maximum of 27.3 years). Procedural and clinical information was extracted and entered into a database for analysis. Time-related outcomes were modeled using multiphase hazard domain techniques. ${ }^{6}$ AVF longevity involved analyzing the time to "AVF occlusion" (point of surgical division/ligation or known spontaneous occlusion). Competing risks methodology ${ }^{7}$ was used to account for patients who died with a functioning AVF and were therefore no longer at risk of AVF occlusion. Separate time-related hazard functions are combined to yield the proportion of children reaching the defined transitional states at any given point in time. Parametric models were then subjected to univariate risk-hazard analysis to identify patient-specific features that influence the risk of (1) AVF occlusion, (2) death with a functioning AVF in situ, and (3) all-cause mortality. Missing values were imputed with the mean for that variable, and the number of missing values is given. Descriptive statistics are presented as frequency with percentage of nonmissing values or median with range. Preoperative and postoperative comparisons of continuous variables were made using paired $t$ tests. All analyses were undertaken using SAS statistical software (SAS Institute Inc, Cary, NC).

\section{RESULTS}

The cohort characteristics are shown in Table 1. The patients were evenly distributed throughout the decades covered by this review (Figure E1). Tricuspid atresia $(\mathrm{N}=8)$ was the most common primary diagnosis. At the time of AVF creation, all patients had previously undergone pallia- tive cavopulmonary shunt (Glenn shunt, $\mathrm{N}=13$; bidirectional cavopulmonary connection, $\mathrm{N}=8$ ), and 5 of these had also undergone total cavopulmonary connection (Fontan completion). AVFs were predominantly right-sided $(86 \%)$ and created at a median age of 13.5 years. The clinical indication for AVF was confirmed pulmonary arteriovenous malformations in 6 patients or otherwise inadequate pulmonary flow and oxygenation in 15 patients. The latter group was a heterogenous case-mix including patients with gradual cyanosis after Glenn in the pre-Fontan era or otherwise specific problems, such as impaired left ventricular function, branch pulmonary artery stenoses interstitial lung disease, and thrombosed great veins (Table E1).

AVFs resulted in a significant improvement in arterial oxygen saturation at all postoperative time points compared with pre-AVF values (Table 1). In addition, last documented New York Heart Association symptom classification (irrespective of subsequent surgical management) was significantly improved compared with pre-AVF values (Table 1).

\section{Outcome of Arteriovenous Fistula}

The time-related fate of the AVF was explored by examining the potential competing outcomes. For all patients, the AVF may continue to function $(\mathrm{N}=6)$ or "occlude" $(\mathrm{N}=10$; no longer provide arteriovenous shunting because of surgical division/ligation or spontaneous thrombosis), or otherwise the patient dies with a functioning $\operatorname{AVF}(\mathrm{N}=5)$. The competing outcomes of these end states for the AVF in the study cohort are shown in Figure 1. Fifteen years after receiving an $\mathrm{AVF}, 30 \% \pm 11 \%$ of the study cohort remained alive with a functioning AVF. In an additional $46 \% \pm 11 \%$ of the study cohort, the AVF was occluded but the patients remained alive, whereas $24 \% \pm 10 \%$ of patients died with a functioning conduit in situ.

\section{Death With a Functioning Arteriovenous Fistula}

Five patients died with a functioning AVF in situ. The type of partial cavopulmonary influenced the risk of death with a functioning AVF. Previous bidirectional cavopulmonary shunt (vs unidirectional Glenn shunt) was associated with a significantly higher time-related risk of death with a functioning AVF in situ, even once adjusted for surgical era (Table 2, Figure 2). The superior survival with a unidirectional Glenn anastomosis was regardless of whether subsequent Fontan had been completed before receiving the AVF (Table 2). In addition, whether the AVF was created after second-stage palliation (cavopulmonary shunt) or Fontan completion did not influence the time-related risk of death with a functioning AVF in situ $(P=.89)$.

An important determinant of patient survival with a functioning AVF was the hemoglobin concentration at the time of AVF creation. Lower hemoglobin levels were associated with significantly worse survival (Figure $3, A)(P<.01)$. The relationship between hemoglobin concentration and 
TABLE 1. Patient characteristics of the study cohort $(N=21)$ of all children at The Hospital for Sick Children who received an arteriovenous fistula for end-stage cyanotic congenital heart disease

\begin{tabular}{|c|c|c|c|}
\hline Characteristic & $\begin{array}{c}\text { Missing } \\
\text { values }\end{array}$ & $\begin{array}{l}\text { Frequency } \\
\text { or median }\end{array}$ & $\%$ or range \\
\hline Male & 0 & 10 & 47 \\
\hline Operative age & 0 & 13.5 & $5 \mathrm{mo}$ to $36 \mathrm{y}$ \\
\hline Left AVF (vs right) & 9 & 2 & 17 \\
\hline \multicolumn{4}{|l|}{ Diagnosis: } \\
\hline Tricuspid atresia & 0 & 8 & 38 \\
\hline Pulmonary atresia & 0 & 3 & 14 \\
\hline Atrioventricular canal defect & 0 & 3 & 14 \\
\hline Double-outlet right ventricle & 0 & 2 & 10 \\
\hline Double-inlet left ventricle & 0 & 2 & 10 \\
\hline $\begin{array}{l}\text { Hypoplastic left heart } \\
\text { syndrome }\end{array}$ & 0 & 2 & 10 \\
\hline $\begin{array}{l}\text { Transposition of the great } \\
\text { arteries }\end{array}$ & 0 & 1 & 5 \\
\hline \multicolumn{4}{|l|}{ Previous CPS: } \\
\hline Glenn CPS & 0 & 13 & 62 \\
\hline Bidirectional CPS & 0 & 8 & 38 \\
\hline Previous Fontan palliation & 0 & 5 & 24 \\
\hline Preoperative $\mathrm{SaO}_{2}(\%)$ & 11 & 74 & $63-90$ \\
\hline $\begin{array}{l}\text { Postoperative } \mathrm{SaO}_{2} \text { in } \\
\text { operating room (\%) }\end{array}$ & 14 & $88^{*}$ & $80-95$ \\
\hline $\begin{array}{l}\text { Postoperative } \mathrm{SaO}_{2} \text { at } \\
\text { discharge }(\%)\end{array}$ & 13 & $81^{*}$ & $75-95$ \\
\hline $\begin{array}{l}\text { Postoperative } \mathrm{SaO}_{2} \text { at latest } \\
\text { follow-up }(\%)\end{array}$ & 12 & $83^{*}$ & $75-90$ \\
\hline Preoperative hemoglobin (g/L) & 11 & 172 & $110-233$ \\
\hline Postoperative hemoglobin (g/L) & 12 & 174 & $139-227$ \\
\hline \multicolumn{4}{|l|}{ Preoperative NYHA class (g/L) } \\
\hline I & 12 & 0 & 0 \\
\hline II & 12 & 3 & 33 \\
\hline III & 12 & 6 & 66 \\
\hline IV & 12 & 0 & 0 \\
\hline \multicolumn{4}{|l|}{ Postoperative NYHA class $\dagger$} \\
\hline I & 11 & 5 & 50 \\
\hline II & 11 & 2 & 20 \\
\hline III & 11 & 3 & 30 \\
\hline IV & 11 & 0 & 0 \\
\hline
\end{tabular}

$A V F$, Arteriovenous fistula; $C P S$, cavopulmonary shunt; $\mathrm{SaO}_{2}$, arterial oxygen saturation; NYHA, New York Heart Association classification. $* P<.01$ vs preoperative value. $\dagger P=.04$ vs preoperative value.

survival was nonlinear, and hemoglobin levels less than 170 $\mathrm{g} / \mathrm{L}$ were associated with particularly bad survival (Figure 3, $B)$. By contrast, the estimated 10 -year risk of death with a functioning AVF was estimated to be $10 \%$ or less if the hemoglobin concentration was more than $185 \mathrm{~g} / \mathrm{L}$ at the time of creating the AVF.

\section{Arteriovenous Fistula Longevity: Time to Occlusion}

The AVF occluded in 10 patients and was ligated during cardiac transplantation in 7 of these patients; alternatively it was removed $(\mathrm{N}=1)$ or spontaneously occluded $(\mathrm{N}=1)$. Risk factors associated with reduced functional longevity

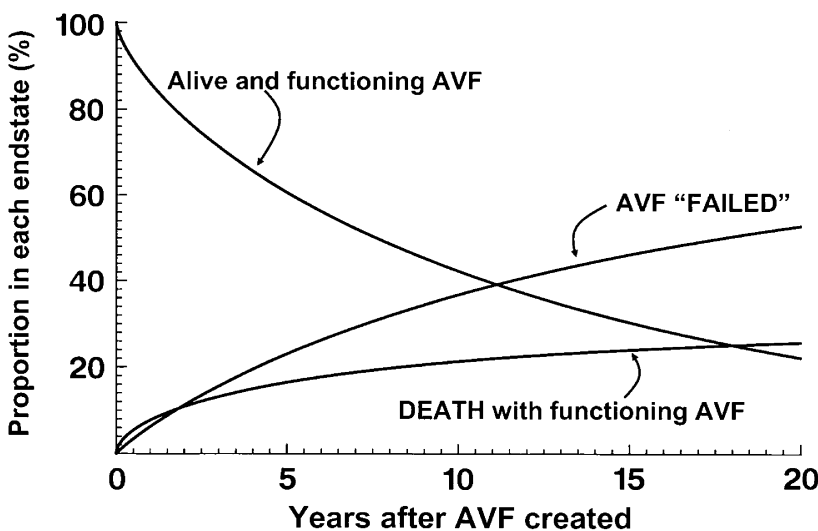

FIGURE 1. Time-related transition to the mutually exclusive competing end states of (1) AVF occlusion, (2) death with a functioning AVF in situ or otherwise, or (3) alive with a functioning AVF in situ. At time zero, all $100 \%$ patients are alive with a functioning AVF. Thereafter, patients assume one of the competing end states at variable rates. Fifteen years after receiving an $\mathrm{AVF}, 44 \% \pm 10 \%$ of patients remain alive with a functioning AVF, $24 \% \pm 8 \%$ of patients have died with a functioning AVF in situ, and $32 \% \pm 11 \%$ of patients had an occluded AVF. The numbers at risk from AVF occlusion were 11,9 , and 3 at 10,15, and 20 years, respectively. Lines represent the parametric determination of continuous point estimates. $A V F$, Arteriovenous fistula.

of the AVF are shown in Table 2. The clinical indication for AVF was a particularly important determinant of outcome. AVFs created for a primary clinical indication of inadequate pulmonary blood flow functioned significantly longer than those created for a primary clinical indication of pulmonary shunting via pulmonary arteriovenous malformations (parameter estimate, $-1.39 ; P=.03$ ). If the AVF was created for an indication of arteriovenous malformations, less than $10 \%$ would be predicted to be alive with a functioning AVF in situ 15 years later (Figure 4, A). By contrast, if the AVF was created because of inadequate pulmonary blood flow, approximately $40 \%$ are estimated to be alive with a functioning AVF in situ 15 years later (Figure 4, $B$ ).

Longevity of AVF function was significantly worse if the AVF was created after Fontan completion versus after second-stage palliation (parameter estimate, $+1.43 ; P=.02$, Figure 5). If the AVF was created after Fontan completion, less than $10 \%$ would be predicted to be alive with a functioning AVF in situ 15 years later (Figure 5, A). By contrast, if the AVF was created after second-stage palliation, approximately $40 \%$ are predicted to be alive with a functioning AVF in situ 15 years later (Figure 5, B).

The use of a Glenn unidirectional cavopulmonary shunt also seemed to offer improved longevity of AVF function (Table 2). Those who received an AVF after Glenn cavopulmonary anastomosis (but before Fontan completion) had significantly better freedom from AVF occlusion $(P=$ $.03)$, although this effect was partly neutralized when changes in surgical era were adjusted for. Neither systemic 
TABLE 2. Univariate associations between patient-specific features and time-related risk of death with a functioning arteriovenous fistula in situ or occlusion of arteriovenous fistula

\begin{tabular}{|c|c|c|c|c|}
\hline \multirow[b]{2}{*}{ Variable } & \multicolumn{2}{|c|}{ Death with functioning AVF $N=5$} & \multicolumn{2}{|c|}{ Occlusion of AVF $N=10$} \\
\hline & Parameter estimate & $P$ value & Parameter estimate & $P$ value \\
\hline Male & - & .16 & - & .22 \\
\hline Operative age (y) & - & .33 & - & 60 \\
\hline Left AVF vs right AVF & - & .11 & - & .65 \\
\hline Tricuspid atresia vs other diagnosis & - & .74 & - & .51 \\
\hline \multirow[t]{2}{*}{ Unidirectional Glenn CPS at time of AVF } & - & .13 & -1.33 & .03 \\
\hline & & & \multicolumn{2}{|c|}{ Adjusted for era* $P=.09$} \\
\hline \multirow[t]{2}{*}{ Unidirectional Glenn CPS, irrespective of subsequent Fontan } & -1.89 & .04 & - & .08 \\
\hline & \multicolumn{2}{|c|}{ Adjusted for era* $P=.05$} & & \\
\hline Previous Fontan completion & - & .89 & +1.43 & .02 \\
\hline Preoperative arterial oxygen saturation $(\%)$ & - & .56 & - & .30 \\
\hline Postoperative arterial oxygen saturations, immediate (\%) & - & .62 & - & .30 \\
\hline Postoperative arterial oxygen saturations at discharge (\%) & - & .95 & - & .21 \\
\hline Preoperative hemoglobin $(\mathrm{g} / \mathrm{dL})$ & -0.07 & $<.01$ & - & .20 \\
\hline Indication: pulmonary blood flow vs pulmonary arteriovenous & - & .87 & -1.39 & .03 \\
\hline
\end{tabular}

malformations

$A V F$, Arteriovenous fistula; $C P S$, cardiopulmonary support. The use of a unidirectional Glenn shunt seemed to confer a general protective influence against both death with a functioning AVF and AVF occlusion. *Interaction terms were explored between the use of unidirectional Glenn cavopulmonary shunts and operative era because increased use of Glenn shunts correlated with earlier operative era (correlation coefficient, $-0.61 ; P<.01$ ).

arterial oxygen saturation at the time of AVF creation nor hemoglobin concentration influenced AVF longevity.

\section{Late Outcomes}

Outcomes after arteriovenous fistula occlusion. Occlusion pertains to cessation of AVF perfusion, and therefore the end of its palliative efficacy. However, a significant portion of the patients in whom the AVF occluded continued to survive. At the close of follow-up (mean, 11 years), 5 of the 9 patients in whom the AVF occluded were still alive. Kaplan-Meier survival estimate for these 9 patients was therefore $73 \% \pm 17 \%$ at 5 years after the AVF occluded.

In 7 of the 9 patients, the AVF occluded when cardiac transplantation was undertaken. The interval from when the AVF was created to the point of transplantation was a median of 4.8 years (range, 4 months to 17 years). Thereafter, survival for these 7 patients was $67 \% \pm 19 \%$ at 5 years from the point of transplantation. Therefore, the AVF acted as a bridge by delaying transplantation approximately 5 years, after which survival was favorable.

Overall late survival. Overall survival for all 21 patients was $73 \% \pm 10 \%, 73 \% \pm 10 \%$, and $37 \% \pm 16 \%$ at 10 , 15 , and 20 years, respectively, from the time of AVF creation (numbers at risk: 13, 6, and 3, respectively). On univariate analysis, no features were identified that significantly influenced overall survival in this cohort. The longest survivor lived for 27.3 years after the AVF was created.

\section{DISCUSSION}

Three quarters of this cohort, who all received an AVF as an end-stage attempt to palliate intractable cyanosis, survived for another 15 years. Although creating an AVF in these circumstances is only rarely considered in contemporary Western practice, this study demonstrates that an AVF remains a useful management option. Their performance was most favorable after second-stage palliation, particularly where cyanosis was caused by suboptimal cavopulmonary shunt flow. In these children, AVF may defer the need for subsequent further surgery (by $\sim 5$ years), and the technique therefore represents a useful bridge to cardiac transplantation.

AVF performance was optimal when cyanosis was not attributable to pulmonary arteriovenous malformations. The reasons for this are obscure but may stem from the fact that intraparenchymal shunting is not addressed by the $\mathrm{AVF}$, and therefore the underlying defect persists (and may progress) after the AVF is created. Instead, in the absence of pulmonary arteriovenous malformations, cyanosis is likely attributable to an overall inadequate pulmonary blood flow (whether secondary to poor cardiac output, low Qp/Qs, or branch pulmonary artery stenoses), and therefore creating an AVF more directly addresses the problem by augmenting pulmonary perfusion pressure to yield a sustained improvement in lung perfusion and oxygenation.

By contrast, the presence of intrapulmonary shunting via arteriovenous malformations is associated with poor performance of AVF. The mechanisms of pulmonary arteriovenous malformations are not fully clarified. Pulmonary arteriovenous malformations originally were attributed to lower lobe perfusion deficits arising from nonpulsatile flow of unidirectional Glenn shunts ${ }^{8}$ in a time-dependent manner. ${ }^{9}$ However, other cavopulmonary anastomoses with nonpulsatile flow are not associated with high risk of 

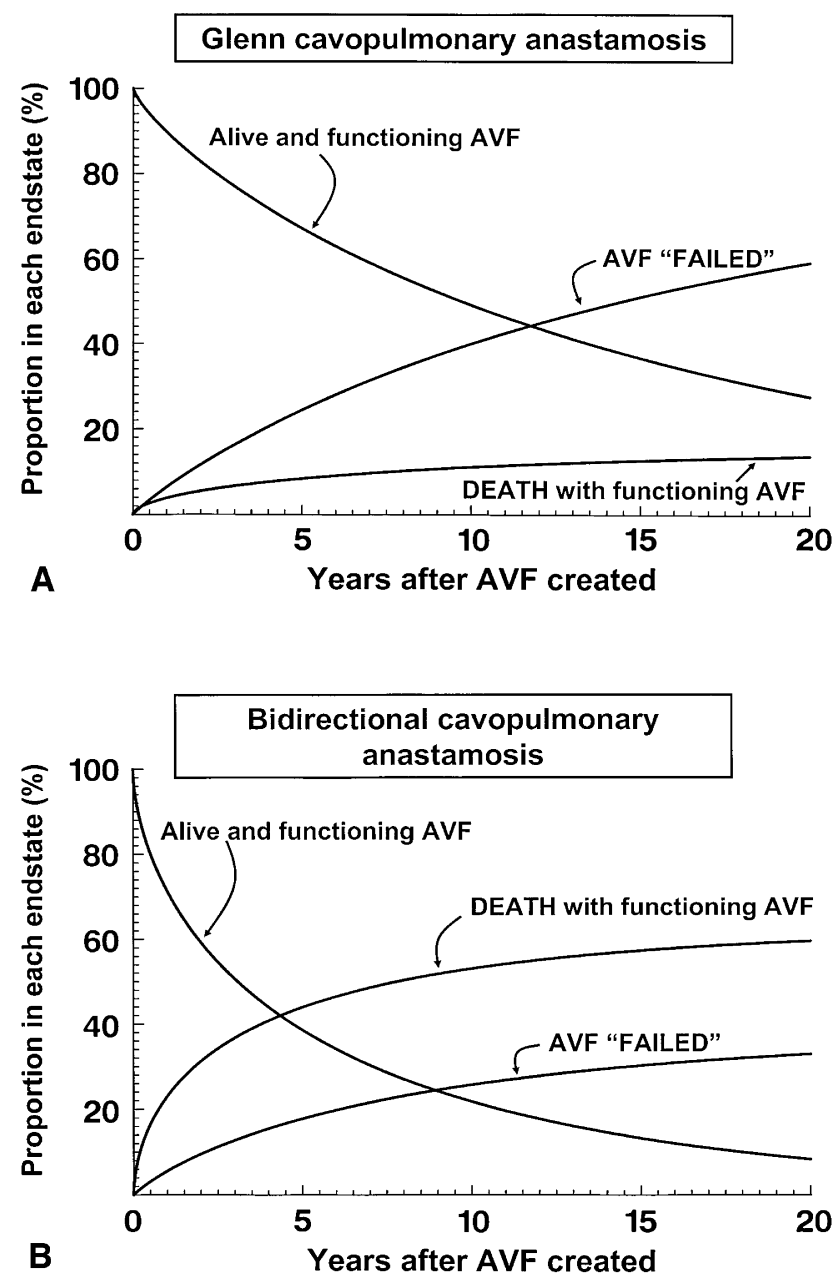

FIGURE 2. Time-related competing outcomes after receiving an AVF, stratified by having undergone Glenn cavopulmonary anastomosis (A) or bidirectional cavopulmonary anastomosis (B). Glenn anastomosis confers a significant protective effect against death with a functioning AVF in situ (parameter estimate, $-1.7 ; P=.01$ ). To adjust for era-dependent changes in surgical practice, the interaction between Glenn anastomosis and operative era was explored as a risk factor and was also significant (parameter estimate, $-0.06 ; P=.04$ ). In those who received a Glenn anastomosis, $18 \% \pm 10 \%$ were predicted to have died with a functioning AVF within 15 years. By contrast, the risk of death with a functioning AVF was $63 \% \pm$ $20 \%$ at 15 years if bidirectional cavopulmonary anastomosis had previously been undertaken. Lines represent parametric determination of continuous point estimates. $A V F$, Arteriovenous fistula.

pulmonary arteriovenous malformations, ${ }^{4}$ and therefore alternative neuroendocrine hypotheses have gained recent interest. Congenital lesions in which hepatoenteric effluent is excluded from the pulmonary circulation are associated with pulmonary arteriovenous malformations, which may reverse once normal anatomic drainage is restored. ${ }^{5}$ In addition, patients with inferior vena cava interruption demonstrate resolution of pulmonary arteriovenous malformations once hepatic drainage is included in the cavopulmonary circulation. ${ }^{10,11}$ Both acute and chronic liver failure in other settings are also known to
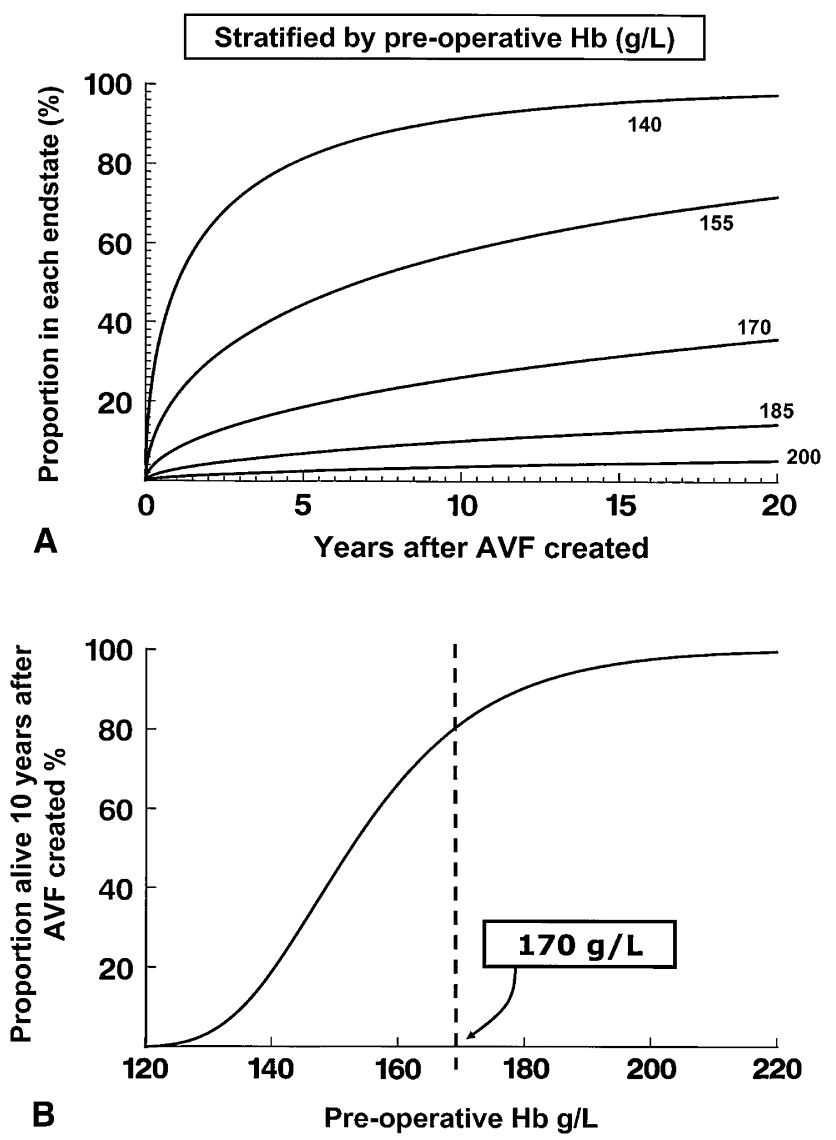

FIGURE 3. A, Time-related risk of death with a functioning AVF in situ, stratified by the circulating hemoglobin concentration at the time of AVF creation. Lower hemoglobin concentrations were associated with significantly worse survival (parameter estimate, $0.07 ; P<.01$ ). B, Nomogram showing the proportion of patients alive 10 years after receiving an AVF across the spectrum of baseline preoperative hemoglobin concentrations. The relationship was nonlinear: hemoglobin levels less than $170 \mathrm{~g} / \mathrm{L}$ conferred disproportionately high mortality, whereas levels more than $185 \mathrm{~g} / \mathrm{L}$ were associated with 10-year risk of death with a functioning AVF in situ of less than $10 \% \pm 8 \%$. The median hemoglobin concentration in the study cohort was $175 \mathrm{~g} / \mathrm{L}$ (range, $110-230 \mathrm{~g} / \mathrm{L}$ ). Lines represent parametric determination of continuous point estimates. $A V F$, Arteriovenous fistula; $H b$, hemoglobin.

be associated with pulmonary vascular abnormalities (hepatopulmonary syndrome ${ }^{12}$ ). Collectively, these findings strongly implicate an absence of humoral "hepatic factors" in the pathogenesis of pulmonary arteriovenous malformations. ${ }^{13,14}$

Poorer performance of AVF in the presence of pulmonary AVF may therefore relate to the fact that underlying neuroendocrine abnormalities persist. Therefore, although AVF may temporarily improve systemic oxygenation, progression of pulmonary shunting may continue unabated; however, AVF has been reported to initiate regression of pulmonary arteriovenous malformations by McElhinney and colleagues. ${ }^{15}$ The authors note that first-pass pulmonary metabolism of hepatic drainage seems to be a necessary 

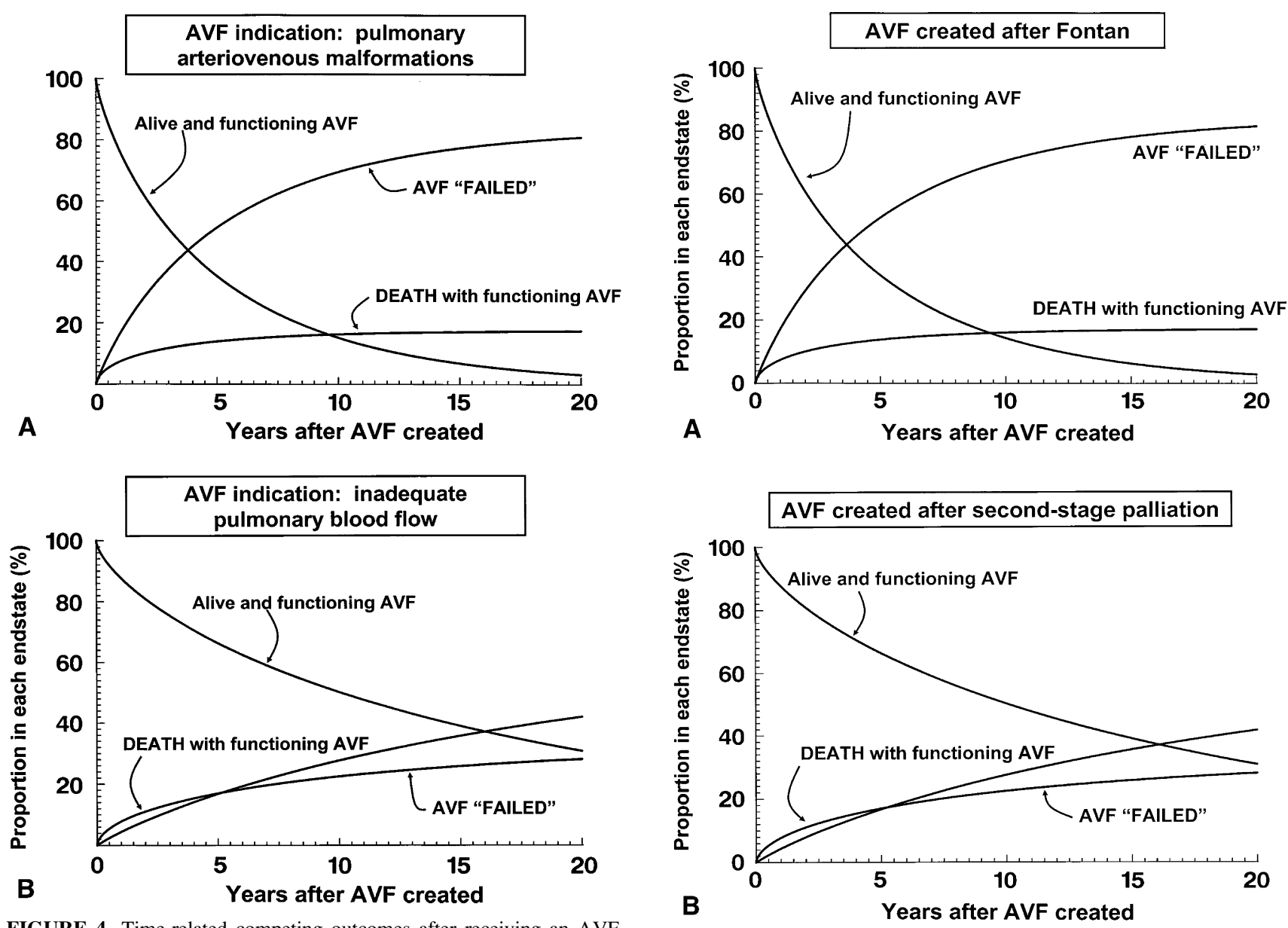

FIGURE 4. Time-related competing outcomes after receiving an AVF, stratified by whether the primary indication was intrapulmonary shunting via arteriovenous malformations (A) or inadequate pulmonary blood flow (B). The rate of AVF occlusion was significantly higher in patients in whom the indication was pulmonary arteriovenous malformations (parameter estimate, $-1.6 ; P=.01$ ). The proportion of AVFs that had occluded at 15 years was $76 \% \pm 18 \%$ (pulmonary arteriovenous malformations) versus $29 \% \pm 12 \%$ (inadequate pulmonary blood flow). Lines represent parametric determination of continuous point estimates. $A V F$, Arteriovenous fistula.

prerequisite for avoiding the development of pulmonary arteriovenous malformations. They postulate that the AVF provides a route for hepatic drainage ejected from the single ventricle to bypass systemic capillary beds and instead perfuse the lung on first-pass, via the cavopulmonary shunt. The interesting concept of a hepatopulmonary neuroendocrine axis may be important in the fetal circulation. Cavopulmonary connection resembles a partial return toward fetal circulation, and pulmonary arteriovenous malformations have been demonstrated in fetal sheep. ${ }^{16}$

An important finding of this investigation is that AVF performance with unidirectional (classic Glenn) cavopulmonary shunts outperformed those with either bidirectional partial cavopulmonary shunts or (bidirectional) total cavopulmonary shunts. A 6- to 7-mm AVF probably will have

FIGURE 5. Time-related competing outcomes after receiving an AVF, stratified by AVF created after Fontan completion (A) or AVF created after second-stage palliation (B). The rate of AVF occlusion was significantly higher in patients in whom Fontan completion had already been undertaken at the time of AVF creation (parameter estimate, $+1.59 ; P=.01$ ). The proportion of AVFs that had occluded at 15 years was $76 \% \pm 17 \%$ (AVF after Fontan) versus $29 \% \pm 11 \%$ (AVF after second-stage palliation). Lines represent parametric determination of continuous point estimates. AVF, Arteriovenous fistula.

a finite maximal flow. Superior performance of AVF with a unidirectional cavopulmonary shunt may be due to concentrating flow to a limited volume of lung tissue. Doubling the downstream lung volume probably dilutes the beneficial effects on cavopulmonary shunt flow.

This analysis examined the outcomes of death with a functioning AVF in situ and AVF occlusion as separate outcomes, rather than treating them as a composite end point. However, both outcomes represent a conceptual clinical "failure" of the AVF. If the composite outcome (of either death or AVF occlusion) is considered, univariate predictors of early composite failure remain: (1) pulmonary arteriovenous malformations $(P=.04)$, (2) previous bidirectional cavopulmonary shunt $(P<.01)$, and $(3)$ AVF after completion Fontan $(P=.05)$. 
An important limitation regarding this study's interpretation for contemporary practice is the age at which AVFs were received. Both in this and previous ${ }^{1,2,15}$ reports, most patients receiving an AVF were young teenagers. It remains to be clarified whether AVF offers comparable performance in young infants with failing cavopulmonary shunts.

\section{CONCLUSIONS}

We reviewed this unique experience to determine the durability of a palliative low-risk option for advanced cyanotic disease. We are not promoting AVF in the current era. However, we suspect that creating an AVF is either an unknown or forgotten option in contemporary practice for patients with severe cyanotic disease. Severe cyanosis from any cause is disabling. It causes severe fatigue and headaches, and may lead to intracerebral thrombosis or hemorrhage. The presence of well-known risk factors may render Fontan completion an unattractive strategy with excessive morbidity and shortened longevity. Some patients, especially adults, and their families may not be willing to accept these outcomes with a high-risk Fontan procedure. Although cardiac transplantation is a theoretic option, in practice these patients are in competition with patients with acute coronary disease and seldom achieve the priority necessary to actually receive a transplant. In either case, an AVF is low risk (potentially an outpatient procedure) and provides relief from the effects of severe cyanosis. Our experience indicates that use of an AVF as a bridge to further management should remain an option in the decision management of the rare patient with intractable cyanosis for whom Fontan completion and transplantation are unappealing.

\section{References}

1. Glenn WW, Fenn JE. Axillary arteriovenous fistula. A means of supplementing blood flow through a cava-pulmonary artery shunt. Circulation. 1972;46:1013-7.
2. Magee A, Sim E, Benson LN, Williams WG, Trusler GA, Freedom RM. Augmentation of pulmonary blood flow with an axillary arteriovenous fistula after a cavopulmonary shunt. J Thorac Cardiovasc Surg. 1996;111:176-80.

3. Cloutier A, Ash JM, Smallhorn JF, Williams WG, Trusler GA, Rowe RD, et al. Abnormal distribution of pulmonary blood flow after the Glenn shunt or Fontan procedure: risk of development of arteriovenous fistulae. Circulation. 1985;72: 471-9

4. Ashrafian H, Swan L. The mechanism of formation of pulmonary arteriovenous malformations associated with the classic Glenn shunt (superior cavopulmonary anastomosis). Heart. 2002;88:639.

5. Lee J, Menkis AH, Rosenberg HC. Reversal of pulmonary arteriovenous malformation after diversion of anomalous hepatic drainage. Ann Thorac Surg. 1998;65: 848-9.

6. Blackstone EH, Naftel DC, Turner MEJ. The decomposition of time-varying hazard into phases, each incorporating a separate stream of concomitant information. J Am Stat Assoc. 1986;81:615-24.

7. Ashburn DA, McCrindle BW, Tchervenkov CI, Jacobs ML, Lofland GK, Bove EL, et al. Outcomes after the Norwood operation in neonates with critical aortic stenosis or aortic valve atresia. J Thorac Cardiovasc Surg. 2003;125: 1070-82.

8. Samanek M, Oppelt A, Kasalicky J, Voriskova M. Distribution of pulmonary blood flow after cavopulmonary anastomosis (Glenn operation). Br Heart J. 1969;31:511-6.

9. Glenn WW, Hellenbrand WE, Henisz A, Laks H, Kope G, Stansel HC, et al. Superior Vena Cava-Right Pulmonary Artery (Glenn) Anastomosis: Present Status. Baltimore: University Park Press; 1984.

10. Shah MJ, Rychik J, Fogel MA, Murphy JD, Jacobs ML. Pulmonary AV malformations after superior cavopulmonary connection: resolution after inclusion of hepatic veins in the pulmonary circulation. Ann Thorac Surg. 1997;63:960-3.

11. Mott AR, Spray TL, Bridges ND. Heart/single-lung transplant for a "failed Fontan'” with pulmonary A-V malformation. Ann Thorac Surg. 1999;67:841-3.

12. Ikai A, Riemer RK, Ma X, Reinhartz O, Hanley FL, Reddy VM. Pulmonary expression of the hepatocyte growth factor receptor c-Met shifts from medial to intimal layer after cavopulmonary anastomosis. J Thorac Cardiovasc Surg. 2004;127:1442-9.

13. Malhotra SP, Reddy VM, Thelitz S, He YP, Hanley FL, Suleman S, et al. Cavopulmonary anastomosis induces pulmonary expression of the angiotensin II receptor family. J Thorac Cardiovasc Surg. 2002;123:655-60.

14. Urcelay GE, Borzutzky AJ, Becker PA, Castillo ME. Nitric oxide in pulmonary arteriovenous malformations and Fontan procedure. Ann Thorac Surg. 2005;80: 338-40.

15. McElhinney DB, Marshall AC, Lang P, Lock JE, Mayer JE Jr. Creation of a brachial arteriovenous fistula for treatment of pulmonary arteriovenous malformations after cavopulmonary anastomosis. Ann Thorac Surg. 2005;80:1604-9.

16. McMullan DM, Hanley FL, Cohen GA, Portman MA, Riemer RK. Pulmonary arteriovenous shunting in the normal fetal lung. J Am Coll Cardiol. 2004;44: $1497-500$. 


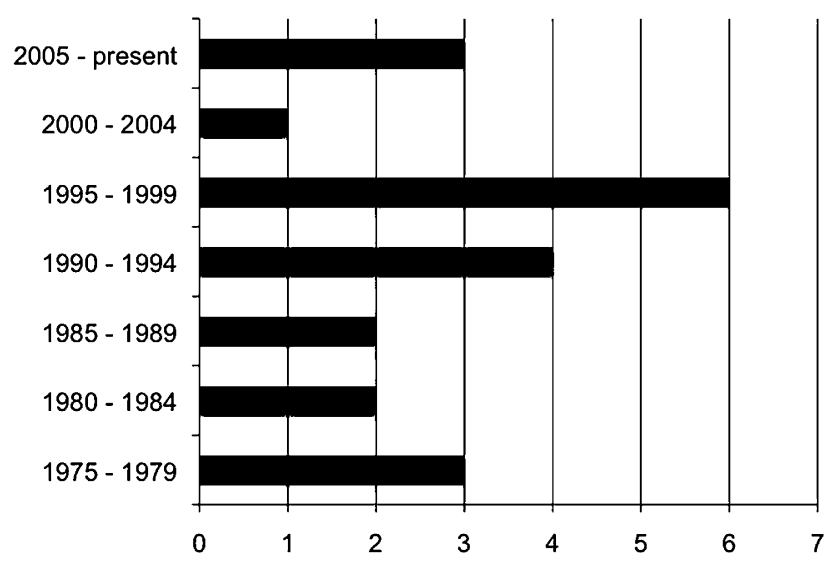

FIGURE E1. Histogram illustrating the number of patients receiving an AVF in each 5-year period from 1975. The date pertains to the date at which the AVF was created.
TABLE E1. Clinical history leading to decision to pursue systemic arteriovenous fistula to manage cyanosis

History leading to AVF

1 Previous Pott's shunt with pulmonary hypertension

2 Hypoplastic left PA; judged not candidate for Fontan

3 Pre-Fontan; Glenn and Pott's shunts with gradual cyanosis

4 Pre-Fontan; Glenn and Pott's shunts with gradual cyanosis

5 Pre-Fontan; Glenn and Pott's shunts with gradual cyanosis

6 LPA stenosis after Pott's shunt; Fontan failed.

7 Previous repair of nonconfluent PAs; Fontan failed.

8 Branch PA stenoses with stents; LPA hypoplasia

9 Interstitial restrictive lung disease; numerous large venous thromboses

10 Poor cavopulmonary shunt flow secondary to innominate vein thrombosis

11 Gradual cyanosis with elevated LV end-diastolic pressures; not Fontan candidate

12 Branch PA stenoses; not Fontan candidate

13 Poor LV function; not Fontan candidate

14 Poor LV function; not Fontan candidate

15 Poor LV function; not Fontan candidate

16 Pulmonary arteriovenous malformations

17 Pulmonary arteriovenous malformations

18 Pulmonary arteriovenous malformations

19 Pulmonary arteriovenous malformations

20 Pulmonary arteriovenous malformations

21 Pulmonary arteriovenous malformations

$A V F$, Arteriovenous fistula; $P A$, pulmonary artery; $L P A$, left pulmonary artery; $L V$, left ventricular. Six patients had pulmonary arteriovenous malformations confirmed by cardiac catheterization. The remaining 15 patients had no evidence of pulmonary arteriovenous malformations on cardiac catheterization. 